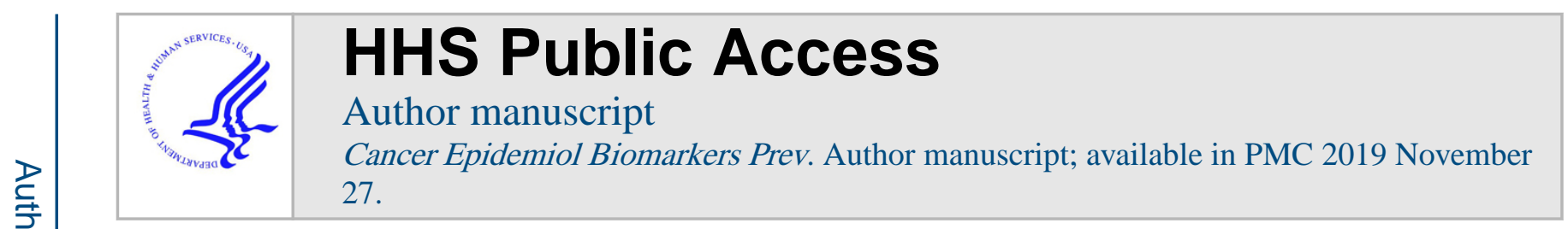

Published in final edited form as:

Cancer Epidemiol Biomarkers Prev. 2010 January ; 19(1): 1-8. doi:10.1158/1055-9965.EPI-08-1111.

\title{
Prostate Cancer Risk Allele Specific for African Descent Associates with Pathologic Stage at Prostatectomy
}

\author{
Eric J. Whitman ${ }^{1}$, Mark Pomerantz ${ }^{4}$, Yongmei Chen ${ }^{3}$, Michael M. Chamberlin ${ }^{1}$, Bungo \\ Furusato $^{2,3}$, Chunling Gao ${ }^{3}$, Amina Ali ${ }^{1}$, Lakshmi Ravindranath ${ }^{3}$, Albert Dobi ${ }^{3,5}$, Isabell A. \\ Sestrehenn $^{2}$, David G. McLeod ${ }^{1,3,5}$, Shiv Srivastava ${ }^{3,5}$, Matthew Freedman ${ }^{4}$, Gyorgy \\ Petrovics $^{3}$
}

${ }^{1}$ Urology Service, Department of Surgery, Walter Reed Army Medical Center ${ }^{2}$ Armed Forces Institute of Pathology, Washington, District of Columbia ${ }^{3}$ Department of Surgery, Center for Prostate Disease Research, Uniformed Services University of the Health Sciences, Rockville, Maryland ${ }^{4}$ Dana Farber Cancer Research Institute, Harvard Medical School, Boston, Massachusetts ${ }^{5}$ U.S. Military Cancer Institute, Uniformed Services University of the Health Sciences, Bethesda, Maryland

\begin{abstract}
Purpose: A region on chromosome 8q24 was recently identified as a novel prostate cancer risk locus. Inherited variation in this region is associated with prostate cancer risk in the general population (21-58\%), and specific alleles show a strong association in African-American men. This study was designed to evaluate associations between $8 \mathrm{q} 24$ risk alleles and clinical variables, such as pathologic stage, age at diagnosis, and recurrence, in a case series of African-American men.
\end{abstract}

Experimental Design: Peripheral blood DNA samples from 114 African-American men with prostate cancer, including 106 who had undergone radical prostatectomy, were genotyped for six single-nucleotide polymorphisms on three $8 \mathrm{q} 24$ regions. The presence of these single-nucleotide polymorphisms was compared with clinicopathologic and follow-up data after radical prostatectomy.

Results: The mean age of diagnosis and follow-up time were $57.4( \pm 8.9)$ years and $49.1( \pm 31.6)$ months, respectively. Patients carrying the Broad11934905 A risk allele, which is specific for African ancestry, were more likely to have a higher pathologic stage $\left(\mathrm{pT}_{3-4}\right)$ than individuals with the wild type (odds ratio, 4.48; 95\% confidence interval, $1.42-14.14 ; P=0.011$ ). A trend toward

\footnotetext{
Corresponding Author: Matthew Freedman, Massachusetts General Hospital, Harvard Medical School, Boston, MA, 02115. Phone: 617-582-8598; Fax: 617-582-8157. freedman@broad.mit.edu or Gyorgy Petrovics, Department of Surgery, Center for Prostate Disease Research, Uniformed Services University of the Health Sciences, Rockville, MD 20852. Phone: 240-453-8942; Fax: 240-453-8912. gpetrovics@cpdr.org.

SupplementarydataforthisarticleareavailableatCancerEpidemiology, Biomarkers and Prevention Online (http://cebp.aacrjournals.org/). The views expressed in this article are those of the authors and do not reflect the official policy of the Department of the Army, Department of Defense, or the U.S. government.

Disclosure of Potential Conflicts of Interest

No potential conflicts of interest were disclosed.
} 
increased frequency of and shorter time to biochemical recurrence was noted in patients with this risk allele on Kaplan-Meier unadjusted survival analysis $(P=0.076)$.

Conclusions: The Broad1 1934905 polymorphism at 8q24, which is only found in people of African ancestry, is associated with an increase in non-organ-confined prostate cancer at prostatectomy. In addition, for those with this risk allele, there is a trend toward early biochemical recurrence that requires validation in larger studies.

\section{Introduction}

Prostate cancer, similarly to other common malignancies, displays health disparities reflecting differences in cancer incidence, mortality, and prevalence among different populations. The most likely reasons for the disparities are due to complex interactions of nonmodifiable (that is, genetic susceptibility, aging) and modifiable risk factors (that is, diet, socioeconomic conditions, health care systems; ref. 1). African-American men have the highest incidence and mortality from prostate cancer in the world and have worse prognosis when compared with Caucasian Americans, with a 1.6-fold increase in chance of being diagnosed and 2.5-fold greater chance of dying from the disease $(2,3)$. Despite evidence that efforts have been made to reverse inequalities in access to health care, including increased use of prostate-specific antigen screening (4), investigators have shown no narrowing of the disparity gap between races (5) and continue to report worse prognostic indices, for example, increased biochemical recurrence, in African Americans (6). In addition to examining socioeconomic factors that may be contributing to these findings, many researchers have been searching for inherited genetics that may help to explain the increased risk for prostate cancer and worse prognosis in men of African ancestry, with some exciting recent findings of single-nucleotide polymorphisms (refs. 7-12).

Despite family history being a known risk factor for prostate cancer, germline risk variants remained elusive until recently (13). In 2006, a risk allele was discovered on the 8q24 gene locus by Amundadottir et al. (7) in an Icelandic population and replicated in an AfricanAmerican population in Michigan. This locus was independently discovered the same year by Freedman et al. using admixture mapping (8). Additional isolation of several 8q24 singlenucleotide polymorphisms associated with prostate cancer risk have been found since then, including one that was present only within the African-American population, the Broad1 1934905 allele of region 2 (10). Although this allele was present at lower frequency (minor allele frequency is $\sim 2 \%$ ), it had the highest odds ratio of all of the markers. Singlenucleotide polymorphisms in African-American men associated with prostate cancer were confirmed by Robbins et al. (14) in 2007, but the Broad allele was not included in that study.

There have been some recent studies linking clinical and pathologic data with risk alleles on $8 \mathrm{q} 24$ in an all Caucasian cohort $(15,16)$ or with a limited number of African Americans (17) but none with a completely African-American cohort and none with biochemical recurrence data. Our hypothesis was that, because several risk alleles at 8q24 differ in frequency between African-American and Caucasian-American men (8), some of the 8q24 variations, especially the ones more frequent in African-American compared with Caucasian-American men, may contribute to the worse prognosis of African-American prostate cancer patients. 
One of these risk alleles, the Broad11934905 single-nucleotide polymorphism, is present only in people with African ancestry (10). Therefore, our primary focus in this study was the analysis of the Broad11934905 allele for association with prostate cancer progression, with emphasis on pathologic stage (extracapsular extension), and prognosis. As a secondary hypothesis, an additional five germline single-nucleotide polymorphisms in three regions of the 8q24 locus have been chosen because they have been shown to be associated with prostate cancer in men of African ancestry (10). These six single-nucleotide polymorphisms, with main emphasis on the Broad1 1934905 single-nucleotide polymorphism, were assessed for associations with comprehensive clinicopathologic findings, including prostatectomy stage and grade, age at diagnosis, and incidence of biochemical recurrence. Specifically, we aimed to identify an allele that may portend the known higher incidence and worse prognosis to this cohort. This is the first study to evaluate the 8q24 risk alleles as they relate to clinical variables in an African-American prostatectomy case series.

\section{Materials and Methods}

From 1995 through 2005, blood samples were collected from 106 African-American men with prostate cancer under Institutional Review Board-approved protocol at Walter Reed Army Medical Center. These patients had undergone radical prostatectomy and had clinical data, pathologic data, and follow-up available. Blinded blood samples were genotyped for six single-nucleotide polymorphisms (rs1447295, rs6983561, rs13254738, Broad11934905, rs6983267, and rs7000448) in three regions of chromosome 8q24 at the Dana Farber Cancer Research Institute.

Radical prostatectomy specimens were all processed and analyzed by genitourinary pathologists at the Armed Forces Institute of Pathology using their standard whole mount technique. Each prostate was formalin fixed, paraffin embedded, and then sectioned at 2.2mm intervals before mounting whole sections on slides (18). Tumor histology was graded using the Gleason grading system, with each tumor being given a Gleason score, and pathologic staging followed the criteria of the American Joint Committee on Cancer standard tumor-node-metastasis annotations. Retrospective review was conducted on clinical records for those patients.

Genotyping was carried out at the Center for Cancer Genome Discovery of the Dana-Farber Cancer Center, using Sequenom iPLEX matrix-assisted laser desorption/ionization-time-offlight mass spectrometry technology. Assays were designed using MassARRAY AssignDesigner application, version 3.0 (Sequenom). PCR and single-base extension oligonucleotides were synthesized by Integrated DNA Technologies. Up to 24 singlenucleotide polymorphism assays were combined into one multiplex pool. All reactions were carried out in 384-well format. PCR amplification was done using an input of $10 \mathrm{ng}$ of DNA in a final volume of $6 \mu \mathrm{L}$. The PCR products were subsequently treated with shrimp alkaline phosphatase, and the single-base extension reactions were initiated using iPLEX enzyme and mass-modified terminators (both Sequenom). After desalting, $7 \mathrm{~nL}$ of the single-base extension reaction was spotted onto a SpectroCHIP (Sequenom) preloaded with $7 \mathrm{~nL}$ of 3hydroxypicolinic acid matrix. The SpectroCHIPs were analyzed in automated mode by a MassArray Compact solid phase laser mass spectrometer system (Bruker Daltonics). The 
resulting spectra were analyzed, and genotypes were called in real time by the SpectroCaller algorithm, followed by additional user-initiated analysis with the SpectroTyper v.3.4A (Sequenom). The error rate on this platform is estimated to be $<0.03 \%$.

Single-nucleotide polymorphisms were assessed for associations with comprehensive clinicopathologic variables. The analyses related to our primary hypothesis included the Broad11934905 single-nucleotide polymorphism association with prostatectomy stage and grade, age at diagnosis, and incidence of biochemical recurrence. Further analyses related to secondary hypothesis assessed the association of all six 8q24 single-nucleotide polymorphisms with the above four clinicopathologic variables. Frequencies were reported for categorical patient features, whereas measures of central tendency and dispersion are reported for continuous patient features. Logistic regression model was adopted to examine the prediction value of Broad1 1934905 single-nucleotide polymorphism on pathologic stage, as well as the prediction value of the other five single-nucleotide polymorphisms.

Differences in age at diagnosis across risk loci or genotype groups were tested using Student's $t$ test or ANOVAs. Risk allele frequency associations with clinicopathologic data (including pathologic T stage, pathologic Gleason score, etc.) were tested using the $\chi^{2}$ test or Fisher's exact test. Kaplan-Meier unadjusted survival analysis and multivariate Cox proportional hazard model were used to examine the relationship of the Broad11934905 single-nucleotide polymorphism, as well as the other five single-nucleotide polymorphisms analyzed, with biochemical recurrence. A two-sided $P<0.05$ was adopted as statistically significant. The statistical software program SAS version 9.1.3 (SAS Institute, Inc.) was used for all data analysis.

\section{Results}

Demographic and clinicopathologic information on the 106 African-American prostate cancer patient study cohort is summarized in Table 1 . This cohort was selected from a total of 253 eligible subjects (African-American prostate cancer patients treated by radical prostatectomy at Walter Reed Army Medical Center between 1995 and 2005) based on availability of blood sample and amount and quality of blood DNA specimens. Of the 253 eligible subjects, 224 had pathologic T-stage data. The participation rate in the $\mathrm{pT}_{2}$ stage group was 55.8\% (63 of 113), and in the $\mathrm{pT}_{3-4}$ stage group, it was $35.1 \%$ (39 of 111). However, the study cohort had no significant difference from the total eligible subject population about prostatectomy pathologic $\mathrm{T}$ stage, as well as grade, age, and biochemical recurrence after radical prostatectomy. The mean age of diagnosis and follow-up time were $57.4( \pm 8.7)$ years and $49.1( \pm 31.6)$ months, respectively. The cohort had $38.2 \%$ patients with high pathologic stage $\left(\mathrm{pT}_{3-4}\right), 13.7 \%$ with high grade (Gleason $\left.8-10\right)$, and $24.8 \%$ with biochemical recurrence after radical prostatectomy (Table 1). The frequencies of the 16 genotypes within the three 8q24 regions studied are shown in Table 2.

As seen in Table 3, the presence of the Broad1 1934905 risk allele is significantly associated with patient's ultimately having non-organ-confined disease $\left(\mathrm{pT}_{3-4}\right)$ at prostatectomy $(P=$ 0.011 ), supporting our primary hypothesis. Moreover, the Kaplan-Meier unadjusted survival analysis shown in Fig. 1 and Table 4 showed a trend toward worse biochemical recurrencefree survival in patients with the Broad11934905 risk allele $(P=0.0762)$. As opposed to the 
Broad1 1934905 risk allele, none of the other five single-nucleotide polymorphisms analyzed associated with pathologic $\mathrm{T}$ stage (Table 3).

To adjust for the important predictors of recurrence we used the Cox proportional hazard model. The Broad risk allele is not significantly associated with recurrence, although it has a hazard ratio of 1.813 , higher than any other single-nucleotide polymorphism analyzed (Table $5)$.

No significant associations were found between biochemical recurrence-free survival and the number of at-risk genotypes per region (Supplementary Figs. S1-S3). None of the individual single-nucleotide polymorphisms showed significant association with postoperative Gleason sum (Supplementary Table S1). Analysis of the total number of at-risk genotypes by region (Supplementary Table S2) did not yield any significant association with clinical or pathologic data, including age at diagnosis or pathologic T stage (Supplementary Tables S3 and S4); however, a trend toward earlier age at diagnosis with increasing number of at-risk genotypes in region 2 is shown in Fig. 2. The total number of at-risk genotypes also did not associate with clinical or pathologic data (Supplementary Table S5; Supplementary Fig. S4).

\section{Discussion}

Genetic association studies have made great strides in discovering common genetic risk alleles underlying prostate cancer risk. To date, most studies have been done on men of European ancestry. However, a large genome-wide admixture study was done on men of African ancestry (8). This study, along with a subsequent fine mapping study, implicated seven risk alleles in the 8q24 region that independently conferred an elevated risk for developing prostate cancer (10). Although these are unequivocally associated with risk for developing prostate cancer, their association with other clinical variables, such as disease aggressiveness, is less well understood. The clinical utility of these markers must be evaluated in the context of clinicopathologic characteristics so that their impact on clinical decision making can be robustly evaluated.

Studies evaluating the relationship of 8q24 inherited polymorphisms to clinical variables have reported variable conclusions. Some studies point to some 8q24 variants conferring an elevated risk for aggressive disease $(7,10,16,17,19,20)$, whereas others do not find the same relationship $(11,14,15,21-24)$. Variation in results between these studies could be due to different populations, different sample sizes, different definitions of aggressive disease, and differential ascertainment. Of note, two recent studies looking at the primary endpoint of prostate mortality do not find association between 8q24 variants and mortality $(25,26)$.

This study comprehensively evaluated the 8q24 risk alleles with clinical factors in a uniformly ascertained African-American prostate cancer population. The most important finding in this study is that of the association of the Broad1 1934905 risk allele with worse clinical stage and a trend toward worse prostate-specific antigen recurrence-free survival. As noted in the Introduction, this single-nucleotide polymorphism is only present in individuals of African ancestry. It is a relatively rare allele yet has the highest magnitude of risk. This 
finding may help to explain some of the disparities observed in African-American men with prostate cancer.

This is a single-institution study that is limited by its small size, but it has use as the first report with comprehensive pathology and follow-up data after prostatectomy in a population of African Americans. Sixteen genotypes of six single-nucleotide polymorphisms in three regions of chromosome 8q24 have been analyzed for association with clinically significant risk factors: age, family history of prostate cancer, tumor grade, and pathologic $\mathrm{T}$ stage. A uniqueness of this African American cohort is that these patients were treated by radical prostatectomy within the equal access Department of Defense healthcare beneficiary system at Walter Reed Army Medical Center.

Most of the studies done to date have extensive data on men of European ancestry. Understanding how or if polymorphisms have differential impacts across populations remains an important and unanswered question and has important implications for delivering personalized medicine.

Helfand et al. recently reported association of $8 \mathrm{q} 24$ susceptibility alleles with tumor characteristics in a Caucasian cohort. Specifically, they found that those with the -8 allele had a significantly greater risk for having high-grade disease (16). Our study is the first to evaluate an African-American cohort for similar associations. The findings presented here suggest that $8 \mathrm{q} 24$ polymorphisms may be associated with worse prognosis.

Currently, it is unknown how this single-nucleotide polymorphism may be associated with disease aggressiveness. The Broad1 1934905 risk allele is located in a region of chromosome $8 \mathrm{q} 24$ with no known genes. The closest known oncogene is c-myc. We can only speculate that this single-nucleotide polymorphism is in an enhancer or suppressor region affecting the expression of either c-myc or another prostate cancer-related gene further away that increases the aggressiveness of prostate cancer.

In conclusion, the evaluation of risk alleles at 8q24 reveals that the Broad11934905 AG allele in African-American prostate cancer patients is associated with non-organ-confined disease. This is the first study to reveal prognostic potential of an 8q24 single-nucleotide polymorphism in African-American prostate cancer patients. Further study on singlenucleotide polymorphisms in larger cohorts is warranted because they show promise as possible markers of aggressive disease.

\section{Supplementary Material}

Refer to Web version on PubMed Central for supplementary material.

\section{Acknowledgments}

The costs of publication of this article were defrayed in part by the payment of page charges. This article must therefore be hereby marked advertisement in accordance with 18 U.S.C. Section 1734 solely to indicate this fact. 


\section{References}

1. Kamangar F, Dores GM, Anderson WF. Patterns of cancer incidence, mortality, and prevalence across five continents: defining priorities to reduce cancer disparities in different geographic regions of the world. J Clin Oncol 2006;24:2137-50. [PubMed: 16682732]

2. Jemal A, Siegel R, Ward E, et al. Cancer statistics, 2008. CA Cancer J Clin 2008;58:71-96. [PubMed: 18287387]

3. Freedland SJ, Isaacs WB. Explaining racial differences in prostate cancer in the United States: sociology or biology? Prostate 2005;62:243-52. [PubMed: 15389726]

4. Ross LE, Berkowitz Z, Ekwueme DU. Use of the prostate-specific antigen test among U.S. men: findings from the 2005 National Health Interview Survey. Cancer Epidemiol Biomarkers Prev 2008;17:636-44. [PubMed: 18349281]

5. Gross CP, Smith BD, Wolf E, Andersen M. Racial disparities in cancer therapy: did the gap narrow between 1992 and 2002? Cancer 2008;112:900-8. [PubMed: 18181101]

6. Schroeck FR, Sun L, Freedland SJ, Jayachandran J, Robertson CN, Moul JW. Race and prostate weight as independent predictors for biochemical recurrence after radical prostatectomy. Prostate Cancer Prostatic Dis 2008;11:371-6. [PubMed: 18427570]

7. Amundadottir LT, Sulem P, Gudmundsson J, et al. A common variant associated with prostate cancer in European and African populations. Nat Genet 2006;38:652-8. [PubMed: 16682969]

8. Freedman ML, Haiman CA, Patterson N, et al. Admixture mapping identifies 8q24 as a prostate cancer risk locus in African-American men. Proc Natl Acad Sci U S A 2006;103:14068-73. [PubMed: 16945910]

9. Gudmundsson J, Sulem P, Manolescu A, et al. Genome-wide association study identifies a second prostate cancer susceptibility variant at 8q24. Nat Genet 2007;39:631-7. [PubMed: 17401366]

10. Haiman CA, Patterson N, Freedman ML, et al. Multiple regions within 8q24 independently affect risk for prostate cancer. Nat Genet 2007; 39:638-44. [PubMed: 17401364]

11. Yeager M, Orr N, Hayes RB, et al. Genome-wide association study of prostate cancer identifies a second risk locus at 8q24. Nat Genet 2007;39:645-9. [PubMed: 17401363]

12. Zheng SL, Sun J, Cheng Y, et al. Association between two unlinked loci at 8q24 and prostate cancer risk among European Americans. J Natl Cancer Inst 2007;99:1525-33. [PubMed: 17925536]

13. Lichtenstein P, Holm NV, Verkasalo PK, et al. Environmental and heritable factors in the causation of cancer-analyses of cohorts of twins from Sweden, Denmark, and Finland. N Engl J Med 2000;343: 78-85. [PubMed: 10891514]

14. Robbins C, Torres JB, Hooker S, et al. Confirmation study of prostate cancer risk variants at 8q24 in African Americans identifies a novel risk locus. Genome Res 2007;17:1717-22. [PubMed: 17978284]

15. Severi G, Hayes VM, Padilla EJ, et al. The common variant rs1447295 on chromosome 8q24 and prostate cancer risk: results from an Australian population-based case-control study. Cancer Epidemiol Biomarkers Prev 2007;16:610-2. [PubMed: 17372260]

16. Helfand BT, Loeb S, Cashy J, et al. Tumor characteristics of carriers and noncarriers of the deCODE 8q24 prostate cancer susceptibility alleles. J Urol 2008;179:2197-201; discussion 202. [PubMed: 18423739]

17. Beebe-Dimmer JL, Levin AM, Ray AM, et al. Chromosome 8q24 markers: risk of early-onset and familial prostate cancer. Int J Cancer 2008;122:2876-9. [PubMed: 18360876]

18. Furusato B, Gao CL, Ravindranath L, et al. Mapping of TMPRSS2ERG fusions in the context of multi-focal prostate cancer. Mod Pathol 2008;21:67-75. [PubMed: 18065961]

19. Suuriniemi M, Agalliu I, Schaid DJ, et al. Confirmation of a positive association between prostate cancer risk and a locus at chromosome 8q24. Cancer Epidemiol Biomarkers Prev 2007;16:809-14. [PubMed: 17416775]

20. Wang L, McDonnell SK, Slusser JP, et al. Two common chromosome 8q24 variants are associated with increased risk for prostate cancer. Cancer Res 2007;67:2944-50. [PubMed: 17409399] 
21. Zheng SL, Sun J, Wiklund F, et al. Cumulative association of five genetic variants with prostate cancer. N Engl J Med 2008;358:910-9. [PubMed: 18199855]

22. Sun J, Chang BL, Isaacs SD, et al. Cumulative effect of five genetic variants on prostate cancer risk in multiple study populations. Prostate 2008;68:1257-62. [PubMed: 18491292]

23. Thomas G, Jacobs KB, Yeager M, et al. Multiple loci identified in a genome-wide association study of prostate cancer. Nat Genet 2008; 40:310-5. [PubMed: 18264096]

24. Schumacher FR, Feigelson HS, Cox DG, et al. A common 8q24 variant in prostate and breast cancer from a large nested case-control study. Cancer Res 2007;67:2951-6. [PubMed: 17409400]

25. Penney KL, Salinas CA, Pomerantz M, et al. Evaluation of 8q24 and 17q risk loci and prostate cancer mortality. Clin Cancer Res 2009.

26. Salinas CA, Koopmeiners JS, Kwon EM, et al. Clinical utility of five genetic variants for predicting prostate cancer risk and mortality. Prostate 2009;69:363-72. [PubMed: 19058137] 


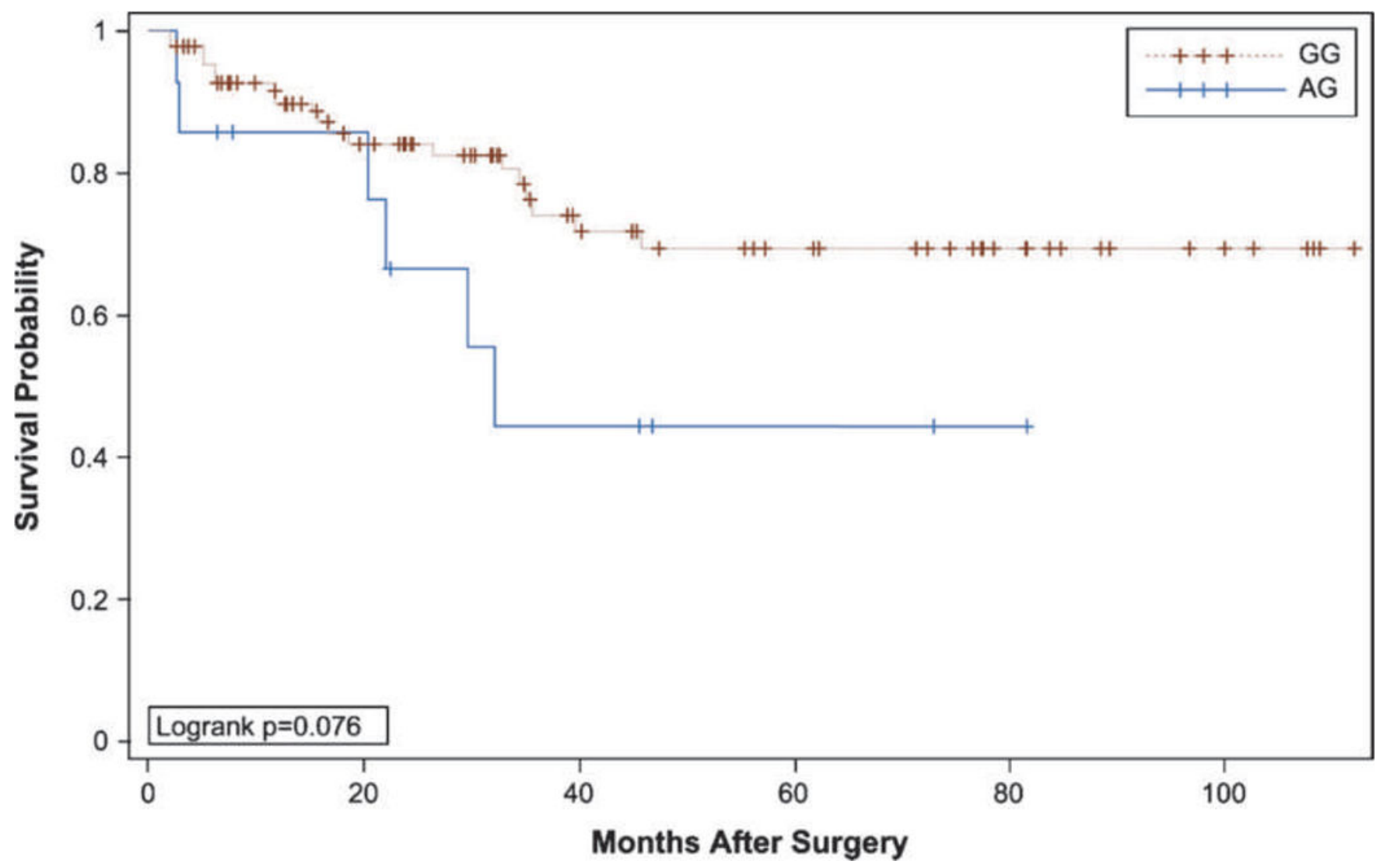

Figure 1.

Kaplan-Meier prostate-specific antigen recurrence-free survival curves with and without the risk allele at the Broad1 1934905 locus of 8q24. GG, wild type; AG, risk allele. 


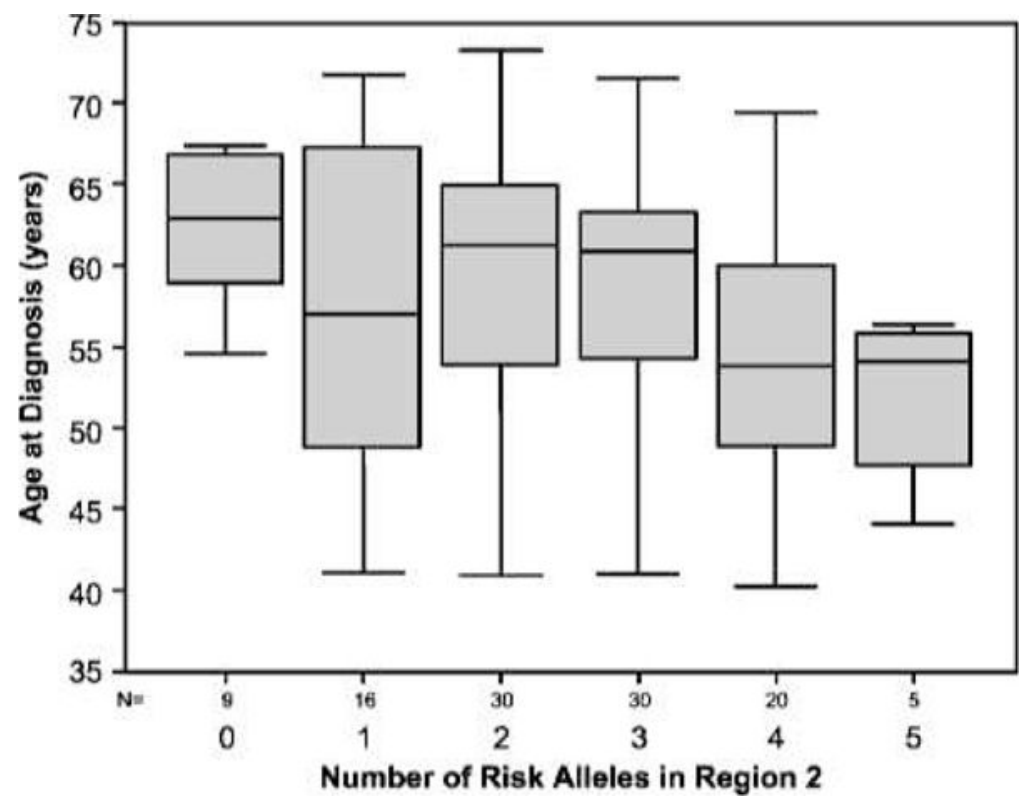

Figure 2.

Association between the number of at-risk genotypes in region 2 and age at diagnosis. For each single-nucleotide polymorphism in region 2, the number of at-risk genotypes was counted. For example, in Rs6983561, wherein the risk allele is $C$, AA is 0; CA, 1; and CC, 2. Then, the number of all at-risk genotypes in region 2 were added up. Although not significant, a trend is observed toward earlier age at diagnosis with more at-risk genotypes. 


\section{롤

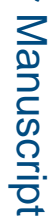
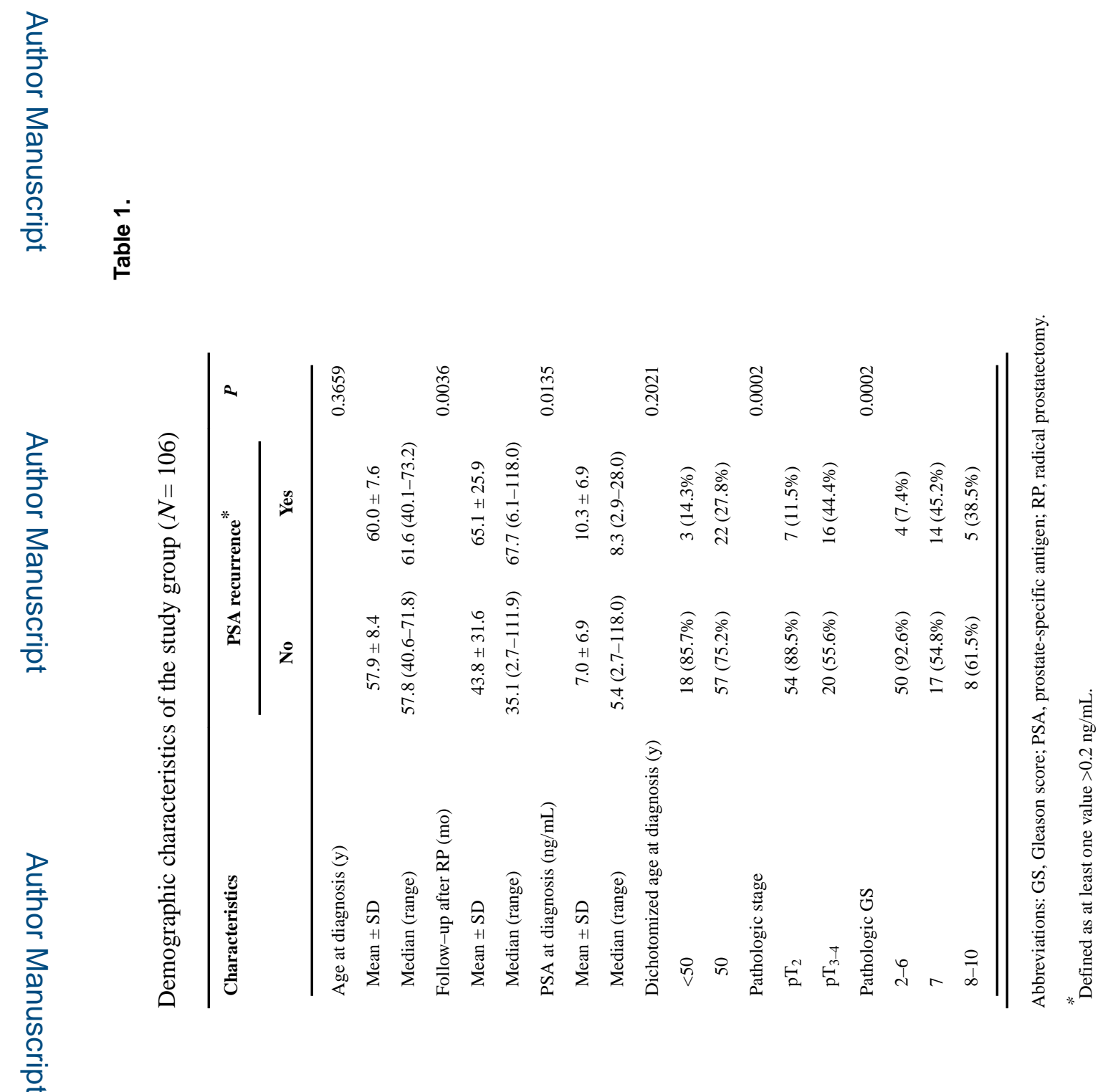

Cancer Epidemiol Biomarkers Prev. Author manuscript; available in PMC 2019 November 27. 


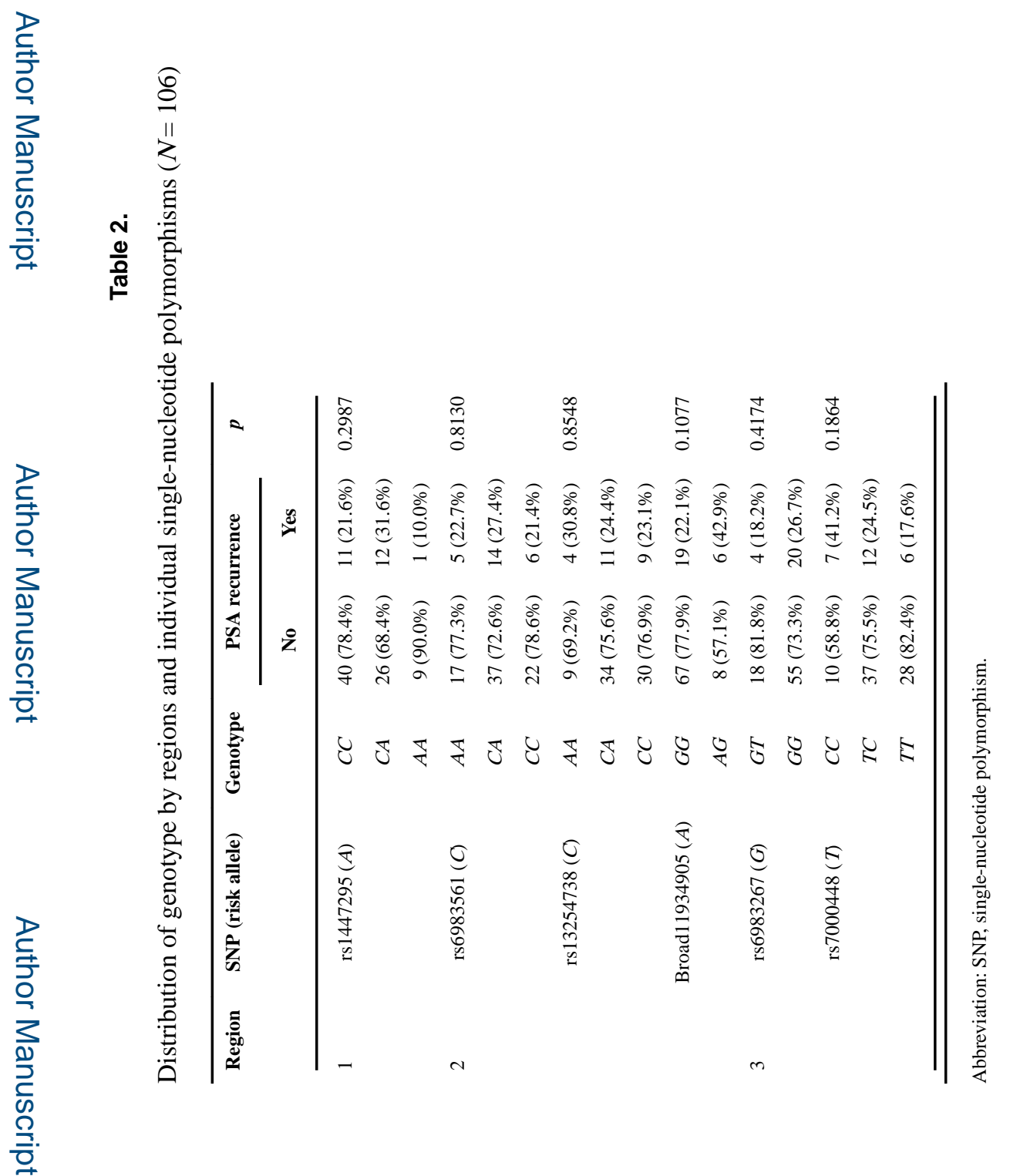




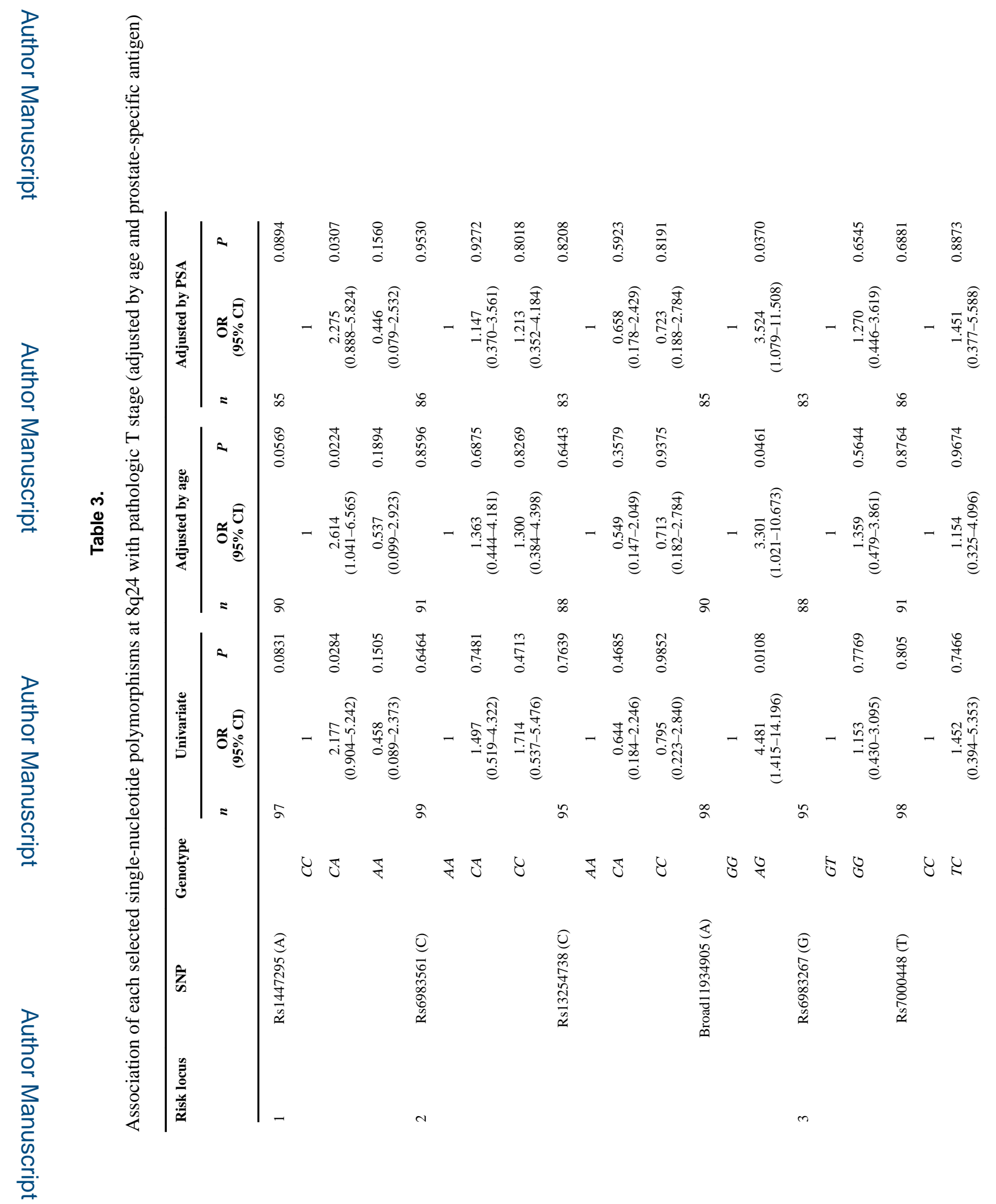




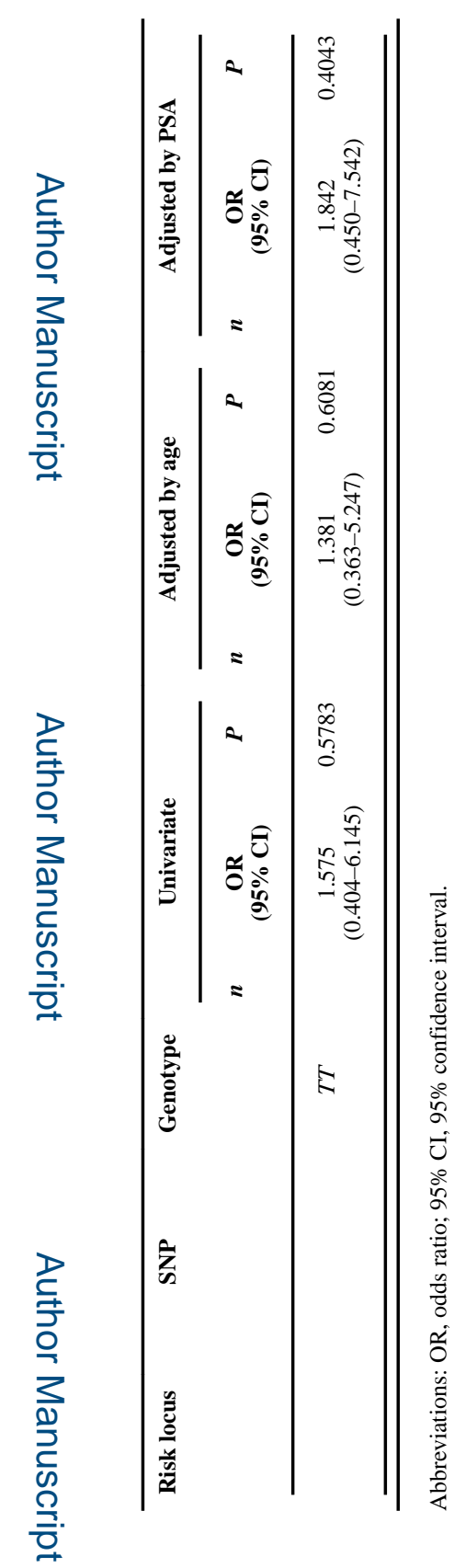



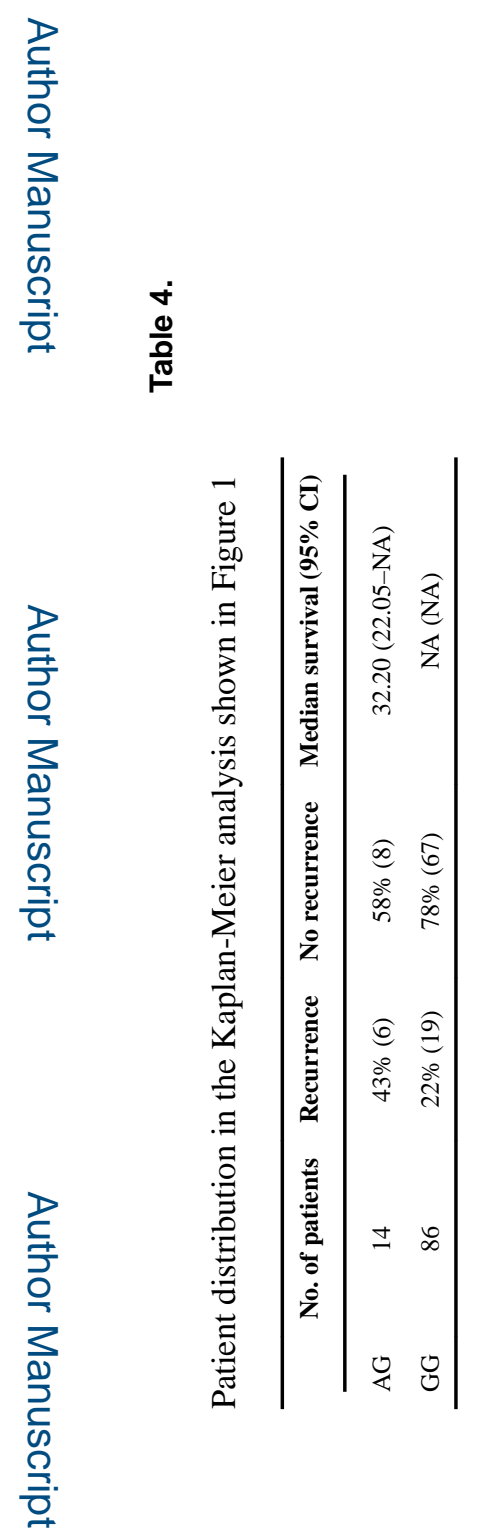


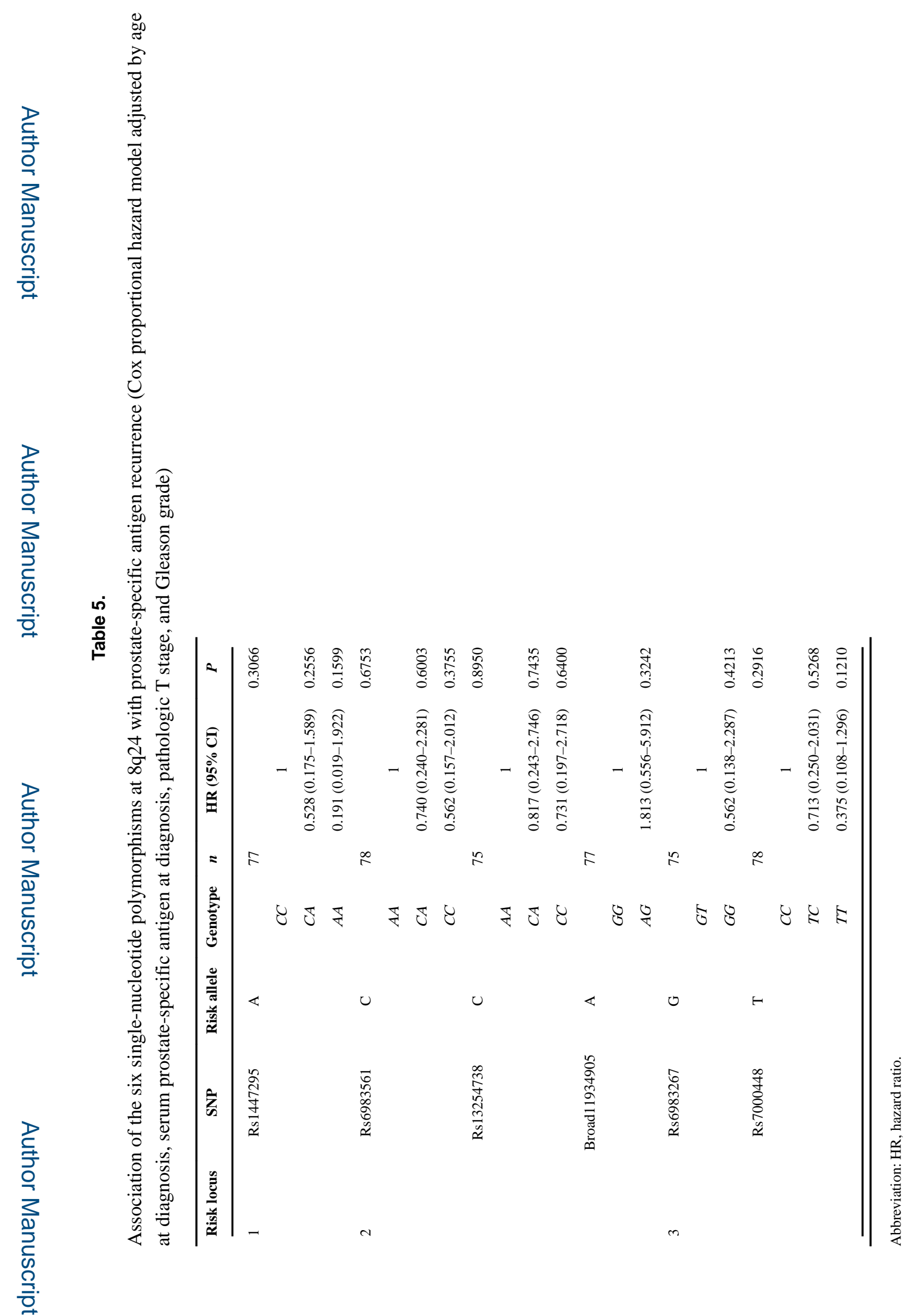

